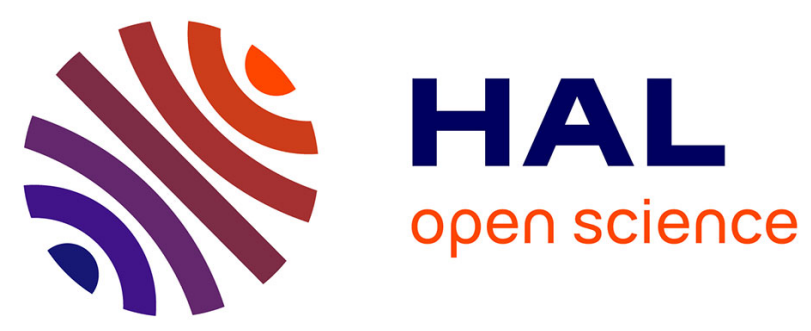

\title{
Effects of the nuclear structure of fission fragments on the high-energy prompt fission $\gamma$-ray spectrum in ${ }^{235} \mathrm{U}\left(n \_\right.$th, $\left.f\right)$
}

H. Makii, K. Nishio, K. Hirose, R. Orlandi, R. Léguillon, T. Ogawa, T. Soldner, U. Köster, A. Pollitt, F.J. Hambsch, et al.

\section{To cite this version:}

H. Makii, K. Nishio, K. Hirose, R. Orlandi, R. Léguillon, et al.. Effects of the nuclear structure of fission fragments on the high-energy prompt fission $\gamma$-ray spectrum in ${ }^{235} \mathrm{U}\left(n \_\right.$th, $\left.f\right)$. Phys.Rev.C, 2019, 100 (4), pp.044610. 10.1103/PhysRevC.100.044610 . hal-02383426

\section{HAL Id: hal-02383426 \\ https://hal.science/hal-02383426}

Submitted on 16 Nov 2020

HAL is a multi-disciplinary open access archive for the deposit and dissemination of scientific research documents, whether they are published or not. The documents may come from teaching and research institutions in France or abroad, or from public or private research centers.
L'archive ouverte pluridisciplinaire HAL, est destinée au dépôt et à la diffusion de documents scientifiques de niveau recherche, publiés ou non, émanant des établissements d'enseignement et de recherche français ou étrangers, des laboratoires publics ou privés. 


\title{
Effects of fission fragment nuclear structure on high-energy prompt fission $\gamma$-ray spectrum in ${ }^{235} \mathbf{U}\left(n_{\mathrm{th}}, f\right)$
}

\author{
H. Makii. * K. Nishio † K. Hirose, R. Orlandi, and R. Léguillon \\ Advanced Science Research Center, Japan Atomic Energy Agency, Tokai, Ibaraki 319-1195, Japan
} states. gence [1-6] after about forty-five years from the elaborated measurements reported in Refs. $7-10$, (see also
Refs. [11-13 ). This is partially due to the requirement orated measurements reported in Refs. $7-10$ (see also
Refs. [11-13]). This is partially due to the requirement to give more precise data for the design of the Gen-IV
fast reactors and advanced light-water reactors [14].

Introduction. In recent years measurements of prompt-fission $\gamma$-ray spectra (PFGS) in low-energy fission processes, such as spontaneous fission (sf) or thermal neutron-induced fission $\left(n_{\mathrm{th}}, f\right)$, experienced a resur- 50

The PFGS carry information on the initial excitation
T. Ogawa

Nuclear Science and Engineering Center, Japan Atomic Energy Agency, Tokai, Ibaraki 319-1195, Japan

T. Soldner, U. Köster, and A. Pollitt

Institut Laue-Langevin, 38000 Grenoble, France

F.-J. Hambsch

European Commission, Joint Research Centre, B-2440 Geel, Belgium

I. Tsekhanovich, M. Aïche, S. Czajkowski, and L. Mathieu

CENBG, CNRS/IN2P3-Université de Bordeaux 19, Chemin du Solarium, 33175 Gradignan, France

C.M. Petrache and A. Astier

Centre de Sciences Nucléaires et de Sciences de la Matière, Université Paris-Sud and CNRS/IN2P3, 91405 Orsay, France

S. Guo

Institute of Modern Physics, Chinese Academy of Sciences, Lanzhou 730000, China and School of Nuclear Science and Technology, University of Chinese Academy of Sciences, Beijing 100049, China

T. Ohtsuki, S. Sekimoto, and K. Takamiya

Institute for Integrated Radiation and Nuclear Science, Kyoto University, Kumatori, Osaka 590-4094, Japan

R.J.W. Frost

Department of Physics, Lund University, Lund S-22100, Sweden

T. Kawano

Theoretical Division, Los Alamos National Laboratory, Los Alamos, New Mexico 87545, USA

The prompt fission $\gamma$-ray energy spectrum for cold-neutron induced fission of ${ }^{235} \mathrm{U}$ was measured in the energy range $E_{\gamma}=0.8-20 \mathrm{MeV}$, by gaining a factor of about $10^{5}$ in statistics compared to the measurements performed so far. The spectrum exhibits local bump structures at $E_{\gamma} \sim 4 \mathrm{MeV}$ and $\sim 6 \mathrm{MeV}$, and also a broad one at $\sim 15 \mathrm{MeV}$. In order to understand the origins of these bumps, the $\gamma$-ray spectra were calculated using a statistical Hauser-Feshbach model, taking into account the de-excitation of all the possible primary fission fragments. It is shown that the bump at $\sim 4 \mathrm{MeV}$ is created by the transitions between the discrete levels in the fragments around ${ }^{132} \mathrm{Sn}$, and the bump at $\sim 6 \mathrm{MeV}$ mostly comes from the complementary light fragments. It is also indicated that a limited number of nuclides, which have high-spin states at low excitation energies, can contribute to the bump structure around $E_{\gamma} \sim 15 \mathrm{MeV}$, induced by the transition feeding into the low-lying high-spin

energy and angular momentum of the primary fission fragments (FFs), important to understand the fission process itself. In low-energy fission processes the average values of the excitation energy vary largely depending on the FFs and are typically about $\bar{E}_{x}=5-25 \mathrm{MeV}$ 15. The average angular momentum of FFs is considered to be $\bar{j}=6-8 \hbar$ [12, 16, 17. Prompt neutrons are dominantly emitted at the first stage of the de-excitation of FFs releasing a large amount of excitation energy, followed by emission of several $\gamma$-rays which remove angu- 
lar momentum. In this simple picture, the maximum $E_{\gamma}$ released is limited to about the neutron binding energy of FF $(5 \sim 7 \mathrm{MeV})$. On the other hand, prompt fission $\gamma$ rays were observed up to energies far larger than $E_{\gamma} \sim 6 \mathrm{MeV}$. The energy reached is $9-10 \mathrm{MeV}$ [2, 3, 18] in low-energy $(n, f)$ reactions and ${ }^{252} \mathrm{Cf}(\mathrm{sf})$ [19 21], indicating that competition between neutron and $\gamma$-ray emissions is evident already at the initial stage of the

${ }_{65}$ de-excitation. For ${ }^{252} \mathrm{Cf}(\mathrm{sf})$, the PFGS were extended as ${ }^{120}$ high as $E_{\gamma} \sim 20 \mathrm{MeV}$ [22 24], with a broad bump structure at $E_{\gamma} \sim 15 \mathrm{MeV}$.

In parallel to the renewal of experimental activities, theoretical calculations of the PFGS using a statistical

70 model are active; see Refs. 25 37. These calculations introduce de-excitation of individual FF, which is benchmarked by the correlation measurement between FFs and prompt neutrons and/or $\gamma$-rays. For the description of $n / \gamma$ emission, information on the structure of neutronhigh excitation energies and discrete levels at low excitation energies are one of the key ingredients to calculate the $n / \gamma$ emission. In the measurement of ${ }^{252} \mathrm{Cf}(\mathrm{sf})$, for example, the PFGS originating from FFs with mass

80 $A \sim 132$ exhibit bump structures at $E_{\gamma} \sim 4 \mathrm{MeV}$ and $^{135}$ $6 \mathrm{MeV}$ [19, 20], which were interpreted by the significantly small level density parameters for FFs in the vicinity of the shell closures at ${ }^{132} \mathrm{Sn}$, obtained from the correlation measurement between FFs and prompt neutrons for ${ }^{252} \mathrm{Cf}(\mathrm{sf}) 38$. Concerning the higher energy bump140 at $E_{\gamma} \sim 15 \mathrm{MeV}$, it was explained by the giant dipole resonance (GDR) $\gamma$-ray emission [23, 24, 39. Although such a $\gamma$-ray emission is allowed only when transitions between the states having similar spin values take place,

90 the calculations did not take into account the low-lying ${ }_{145}$ nuclear level structure of the neutron-rich FFs; thus the relevance between the high-energy $\gamma$-ray emission and the fission fragment nuclear structure was not argued so far.

In this work we extended the measurement of

PFGS for cold-neutron induced fission of ${ }^{235} \mathrm{U}, 150$ ${ }^{235} \mathrm{U}\left(n_{\mathrm{th}}, f\right)$, from $E_{\gamma}=0.8 \mathrm{MeV}$ to about $20 \mathrm{MeV}$, by gaining a factor of about $10^{5}$ in statistics compared to the available data for cold/thermal neutron induced fissions. We observed local bump structures at $E_{\gamma} \sim 4 \mathrm{MeV}$ and $\sim 6 \mathrm{MeV}$, and a broad one at $\sim 15 \mathrm{MeV}$, similar to155 ${ }^{252} \mathrm{Cf}(\mathrm{sf})$. We adopted the most-advanced statistical model calculation [40, where the low-lying nuclear structure of neutron-rich FFs was introduced. Thus more accurate analysis to deduce the origins of the observed structures became possible.

Finally we mention that the high-energy $\gamma$ rays in fission could be used for the decommissioning of the damaged Fukushima atomic power plants in Japan. As they have far higher energies than the background $\gamma$ rays produced in the $\beta$-decay of fission products in the debris, 165 they are easily distinguished from the background $\gamma$ rays, thus might be used to monitor the fission rate for the crit- icality surveillance.

Experimental methods and results. The measurement of the PFGS for ${ }^{235} \mathrm{U}\left(n_{\mathrm{th}}, f\right)$ was carried out at the PF1B cold-neutron beam facility 41 at the Institut Laue-Langevin (ILL), Grenoble, France. Details of the experimental setup and data analysis are explained in Ref. 42, thus only the most pertinent information is given here. A ${ }^{235} \mathrm{U}$ target (99.93\% isotopic enrichment) had $117 \mu \mathrm{g} / \mathrm{cm}^{2}$ in thickness and $30 \mathrm{~mm}$ in diameter, evaporated on a $33 \mu \mathrm{g} / \mathrm{cm}^{2}$ polyimide backing coated by $45 \mu \mathrm{g} / \mathrm{cm}^{2} \mathrm{Au}$. The neutron beam was collimated to $\sim 20$ $\mathrm{mm}$ in diameter and the neutron capture flux adjusted to $1.0 \times 10^{8} \mathrm{n} / \mathrm{cm}^{2} / \mathrm{s}$ at the target position. The experimental setup consists of two position-sensitive multiwire proportional counters (MWPCs) with active area $80 \times 80 \mathrm{~mm}^{2}$, used to detect both FFs in coincidence, and two large volume $\mathrm{LaBr}_{3}(\mathrm{Ce})$ scintillators $(101.6 \mathrm{~mm}$ in diameter and $127.0 \mathrm{~mm}$ in length), located behind each MWPC. The distance between the target-center and the front surface of the scintillator was $280 \mathrm{~mm}$. Throughout the 437 hour run, $5.1 \times 10^{10}$ coincident FF events have been acquired. The total number of observed $\gamma$-rays in coincidence with FFs was $1.7 \times 10^{9}$.

The masses of FFs were obtained event-by-event by measuring the time-of-flight (TOF) difference for both FFs to traverse from the target to each MWPC (the minimum distance is $50 \mathrm{~mm})$. Figure 1(a) shows the fission-fragment mass distributions obtained in the experiment. The mass resolution was 27 amu (FWHM). In order to separate the prompt $\gamma$ rays from the prompt fission neutrons, we also measured the TOF (denoted as $T_{\gamma}$ hereafter) using the FF START signal from the MWPC and the $\gamma$-ray STOP signal from the $\mathrm{LaBr}_{3}(\mathrm{Ce})$ scintillator. Figure 1(b) and (c) show the recorded events on the $T_{\gamma}$ versus pulse height from the scintillator. The spectrum (b) is obtained with a condition that light FFs with mass of $97 \leq A_{\mathrm{L}} \leq 116$ (marked as the region 'A' in Fig. 1(a)) are emitted toward one of the scintillators which generates the signal. Data of Fig. 1 (c) is the same as the spectrum (b), but for heavy FFs with $120 \leq A_{\mathrm{H}} \leq 139$ (region 'B' in Fig. 1(a)). One can notice that the yields associated with prompt neutrons are larger in the spectrum (b) than (c). This is explained by the larger (smaller) neutron multiplicity for the FFs around $A_{\mathrm{L}} \sim 104\left(A_{\mathrm{H}} \sim 132\right)$, see for example Ref. 43 . and Fig. 4(a), and these neutrons are strongly focused to the FF direction in the laboratory frame, where the scintillator is placed. Prompt $\gamma$-ray events are obtained by applying the gate conditions on $T_{\gamma}$ vs pulse-height spectrum (indicated by the areas enclosed by the dashed lines in Fig. 1(b) and (c)). We also subtracted the timeindependent background events entering into the prompt $\gamma$-ray gate. They are produced mostly by the capture of scattered neutrons with various materials around the ${ }^{235} \mathrm{U}$ target, thus observed independently of the $T_{\gamma}$. An 

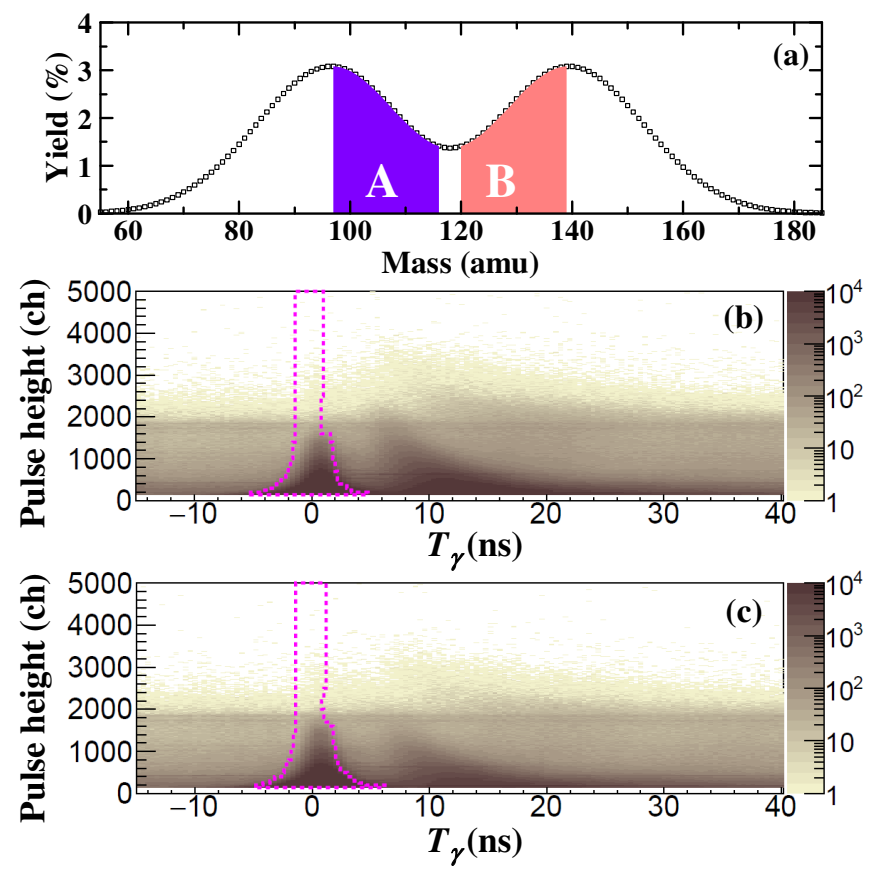

FIG. 1. (a) Fission-fragment mass distribution for ${ }^{235} \mathrm{U}\left(n_{\mathrm{th}}, \mathrm{f}\right)$ obtained from the measurement of the time-offlight difference. (b) Recorded events on the $T_{\gamma}$ vs pulse height plane from the $\mathrm{LaBr}_{3}(\mathrm{Ce})$ scintillator under the condition that fragments contained in the region 'A' of the panel (a) are moving to the scintillator of interest. (c) Same as (b), but for the fragments in 'B'. The areas enclosed by the dashed lines in (b) and (c) indicate the gate position used to select the prompt $\gamma$-ray events.

TABLE I. Average total $\gamma$-ray multiplicity in fission $\bar{M}_{\gamma}$, average $\gamma$-ray energy $\bar{\epsilon}_{\gamma}$, and average total $\gamma$-ray energy $\bar{E}_{\gamma \text {,tot }}$ for ${ }^{235} \mathrm{U}\left(n_{\mathrm{th}}, f\right)$ in the energy range of $1 \leq E_{\gamma} \leq 6 \mathrm{MeV}$.

\begin{tabular}{|c|c|c|c|}
\hline & $\bar{M}_{\gamma}$ & $\bar{\epsilon}_{\gamma}$ & $\bar{E}_{\gamma, \text { tot }}$ \\
\hline Present Expt. & $2.29 \pm 0.05$ & $1.78 \pm 0.05$ & $4.07 \pm 0.07$ \\
\hline Peelle et al. 7$]^{a}$ & $2.48 \pm 0.04$ & $1.82 \pm 0.04$ & $4.51 \pm 0.07$ \\
\hline Verbinski et al. $[9]$ & $2.16 \pm 0.03$ & $1.84 \pm 0.03$ & $3.98 \pm 0.05$ \\
\hline Obersted et al. [4] & $2.29 \pm 0.05$ & $1.86 \pm 0.07$ & $4.23 \pm 0.08$ \\
\hline Present Calc. $\mathrm{CoH}_{3}$ & 2.45 & 1.86 & 4.57 \\
\hline
\end{tabular}

a The quoted uncertainties are statistical ones only.

energy calibration of the scintillator was made by refer- ${ }_{185}$ ring to the $\gamma$ rays from the ${ }^{27} \mathrm{Al}(n, \gamma){ }^{28} \mathrm{Al}$ reaction (3.47, $3.59,5.13,6.10,6.32$, and $7.72 \mathrm{MeV}$ ) and the $\beta$-decay of ${ }^{28} \mathrm{Al}(1.78 \mathrm{MeV})$ in the time-independent background $\gamma$-ray spectrum. An extension of the calibration to the higher energy region at $8 \leq E_{\gamma} \leq 20 \mathrm{MeV}$ was given 190 by referring to the well established response of the scintillator obtained from several $\gamma$ rays $(4.44,8.72,13.92$, and $18.36 \mathrm{MeV})$ from the ${ }^{11} \mathrm{~B}(p, \gamma){ }^{12} \mathrm{C}$ reaction studied at JAEA-Tokai tandem accelerator facility [42].

The final PFGS for ${ }^{235} \mathrm{U}\left(n_{\mathrm{th}}, f\right)$ were deduced by the 195 spectrum unfolding method [42, 45]. Figure 2 shows the

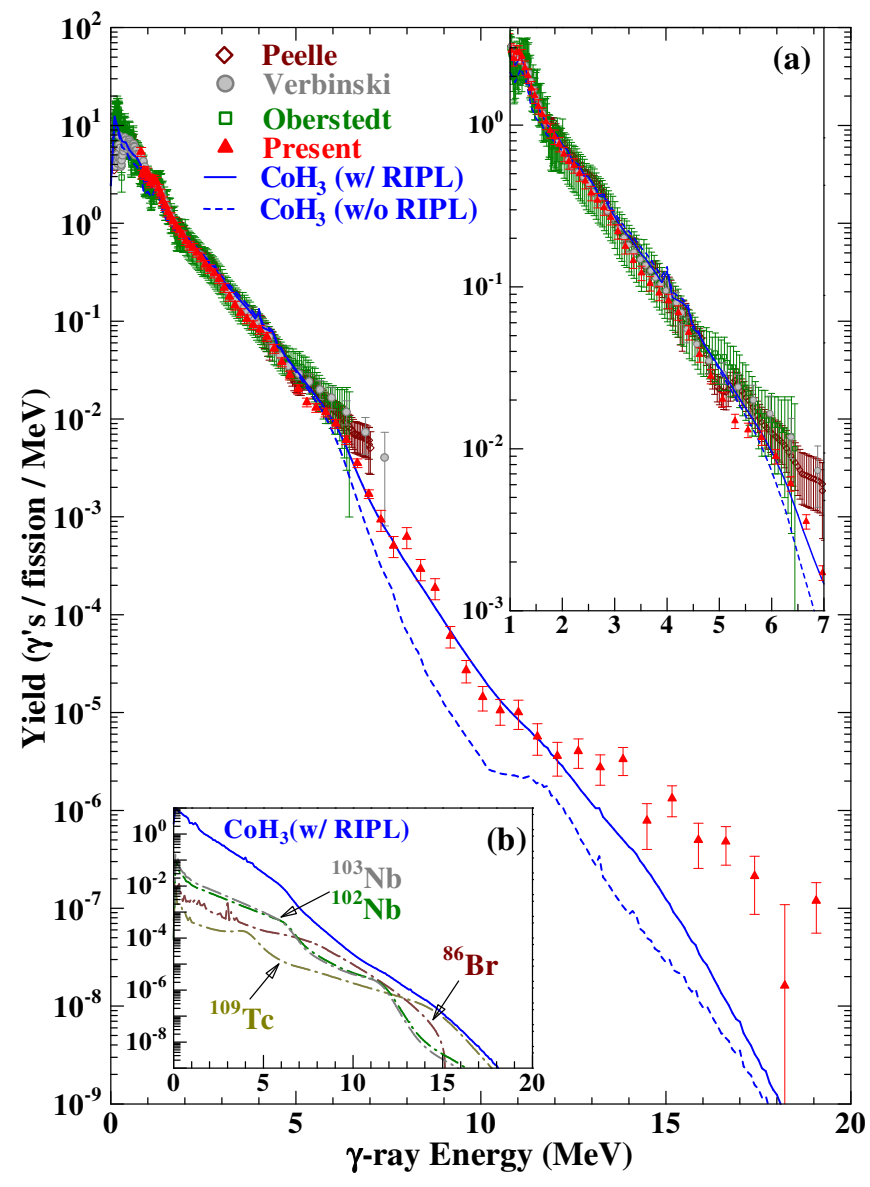

FIG. 2. Prompt $\gamma$-ray spectra obtained in the present study (filled triangles) are compared to those by Peelle et al. (open diamonds) 7], Verbinski et al. (filled circles) 9], and Obersted et al. (open rectangles) 4]. The spectra are compared with the calculation by the $\mathrm{CoH}_{3}$ code [4] (solid and dashed lines); see text for details. Expansion of the spectra in the energy range $E_{\gamma}=1-7 \mathrm{MeV}$ is shown in the inset (a). The inset (b) shows the $\gamma$-ray spectra for ${ }^{86} \mathrm{Br},{ }^{102,103} \mathrm{Nb}$, and ${ }^{109} \mathrm{Tc}$ with the $\mathrm{CoH}_{3}$ code 44] (dashed-dotted lines).

results of the PFGS. The present data significantly increased the statistics by a factor of $\sim 10^{5}$ compared to the literature data [4, 7, 9], and the data points are extended to $E_{\gamma} \sim 20 \mathrm{MeV}$ for the first time in cold/thermal neutron induced fission. Uncertainties in the present data contain statistical error and systematic errors coming from the detection efficiency of the scintillator as well as the prompt $\gamma$-ray gate condition [42]. It is found that the present data are in good agreement with the literature data up to $E_{\gamma} \sim 4.8 \mathrm{MeV}$. Beyond this energy, however, the data in Refs. 4, 7, 9, have large uncertainties. Gained statistics in the present study clearly revealed the local bumps around $E_{\gamma}=4.0$ and $5.8 \mathrm{MeV}$ as shown in the inset (a) of Fig. 2 The observed average properties of the $\gamma$-ray emission (average total $\gamma$-ray multiplicity in fission $\bar{M}_{\gamma}$, average $\gamma$-ray energy $\bar{\epsilon}_{\gamma}$, and average total $\gamma$-ray energy released in fission $\bar{E}_{\gamma, \text { tot }}$ ) are in fairly good 


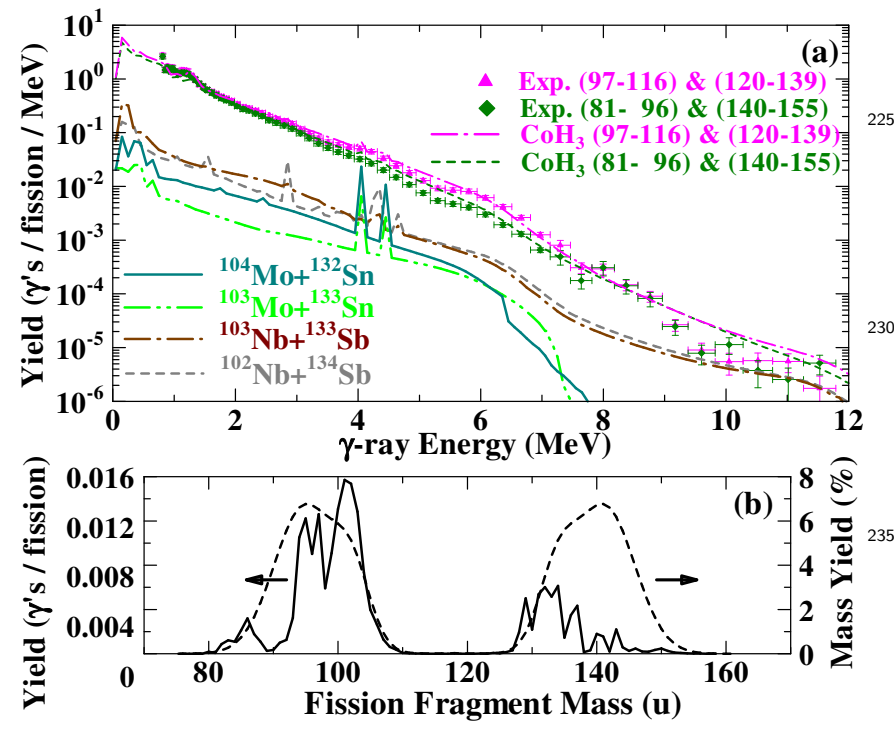

FIG. 3. (a) PFGS obtained for fragment mass splits with $97 \leq A_{\mathrm{L}} \leq 116$ or $120 \leq A_{\mathrm{H}} \leq 139$ (filled triangles) and $_{240}$ $81 \leq A_{\mathrm{L}} \leq 96$ or $140 \leq A_{\mathrm{H}} \leq 155$ (filled diamonds) are compared to the corresponding $\mathrm{CoH}_{3}$ calculations (dashed-dotted line and dashed line). Selected spectra from the calculation having the largest contribution to the bump at $E_{\gamma}=4.0 \mathrm{MeV}$ are shown $\left({ }^{104} \mathrm{Mo}+{ }^{132} \mathrm{Sn},{ }^{103} \mathrm{Mo}+{ }^{133} \mathrm{Sn},{ }^{103} \mathrm{Nb}+{ }^{133} \mathrm{Sb}\right.$, and ${ }^{102} \mathrm{Nb}+{ }^{134} \mathrm{Sb}$ ). (b) Calculated $\gamma$-ray yield in the energy range 245 of $5.3 \leq E_{\gamma} \leq 6.5 \mathrm{MeV}$ as a function of the fragment mass (solid line). The mass yield distribution of Ref. [46] is given by the dashed line.

agreement with those from literature data as summarized in Table I. Above $E_{\gamma}=8 \mathrm{MeV}$ the present PFGS exhibit a broad bump centered around $E_{\gamma}=15 \mathrm{MeV}$, similar to ${ }^{252} \mathrm{Cf}(\mathrm{sf})$ [23, 24].

To facilitate understanding the origins of the local $_{255}$ bumps around $E_{\gamma}=4.0$ and $5.8 \mathrm{MeV}$, we obtained the mass-gated PFGS. Filled triangles and filled diamonds in Fig. 3(a) indicate the PFGS for the fragment mass lits in the "near-symmetric region" $\left(97 \leq A_{\mathrm{L}} \leq 116\right.$ or $\left.120 \leq A_{\mathrm{H}} \leq 139\right)$ and the "large-asymmetric region" ${ }_{260}$ $\left(81 \leq A_{\mathrm{L}} \leq 96\right.$ or $\left.140 \leq A_{\mathrm{H}} \leq 155\right)$, respectively. The PFGS for the near-symmetric region show the more pronounced local bumps around $E_{\gamma}=4.0$ and $5.8 \mathrm{MeV}$, thus tures. The smaller bumps at the same location for the ${ }_{265}$ large-asymmetric region must be due to the limitted mass resolution (FWHM $\sim 27 \mathrm{amu}$ ). Similar structures were observed in ${ }^{252} \mathrm{Cf}(\mathrm{sf})$ where their origin was assigned to

the heavy FFs in the vicinity of $A \sim 132$ [20].

Discussion. In order to understand the origins of the 270 bump structures observed in the PFGS, the statistical Hauser-Feshbach model calculation was carried out using the code $\mathrm{CoH}_{3}$ [40, 44]. In this model, $\gamma$-ray emis-

sion from all the possible primary FFs are introduced,275 which are aggregated over the yield distribution of the FFs given as a function of mass $(A)$, charge $(Z)$, total kinetic energy (TKE), spin $(J)$, and parity $(\pi)$. For details, see Refs. 29, 40, 47]. Instead of using the Monte Carlo technique, we adopted a deterministic method in order to see the spectra with small yield. For the distribution of the primary FFs on $A, Z$, and TKE, we used experimental data [46] complemented by the Wahl systematics for the $Z$ distribution 48 . Sharing of the total excitation energy, obtained by subtracting the TKE from the $Q$-value of the fission reaction, between the FFs was determined by an anisothermal parameter defined as $R_{T}=T_{L} / T_{H}$ using the initial light $\left(T_{L}\right)$ and heavy $\left(T_{H}\right)$ FF temperatures [49 51]. The $J$ and $\pi$ distributions are given by

$$
R(J, \pi)=\frac{J+1 / 2}{2 f^{2} \sigma^{2}(U)} \exp \left\{-\frac{(J+1 / 2)^{2}}{2 f^{2} \sigma^{2}(U)}\right\},
$$

where the $\pi$ distribution is equally probable for even and odd parities 40. $\sigma^{2}(U)$ is the spin cut-off parameter as a function of the effective excitation energy $U$ determined by fragment excitation energy $E_{x}$ and pairing energy $\Delta$ via $U=E_{x}-\Delta$. The variable $f$ is a scaling factor to adjust the spin distribution of the primary FFs. We used the parameters $R_{T}=1.3$ and $f=2.5$, which can reproduce average prompt neutron multiplicity and its fragment-mass dependence $\bar{\nu}(A)$ [40, 47, as shown in Fig. 4(a). The neutron transmission coefficients are given by the global optical potential of Koning and Delaroche [52]. For E1 $\gamma$ rays, the $\gamma$-ray transmission coefficients are calculated with the E1 GDR [53] with the parameters in Ref. [54. In addition, we include the $\gamma$ ray multipolarities of M1, E2, M2, and E3. We also consider the M1 scissors mode for deformed nuclei [55]. We use phenomenological level density formula of GilbertCameron 56 with an updated parameterization 57]. It has the same functional form as the $J$ distribution of the primary FFs as in Eq. (1), and the $\sigma^{2}$ parameter characterizes the spin distribution of the level density. At low excitation energies, $\gamma$-ray transitions between the discrete levels are included. The discrete level data are taken from the Reference Input Parameter Library RIPL3 database [58. Figure 4(b) shows the average $\gamma$-ray multiplicity from each $\mathrm{FF}, \bar{M}_{\gamma}(A)$, as a function of the fragment mass. A relatively good agreement with the literature data [8, 59] was obtained.

The solid lines in Fig. 2 indicate the calculated PFGS using the $\sigma^{2}$ parameters obtained from the spindistribution analysis of the low-lying levels available in the RIPL-3 [58]. For nuclei whose levels are not compiled in the RIPL-3 [58], the parameter following the systematics was adopted. The spectrum agrees well with the present data at $E_{\gamma}<12 \mathrm{MeV}$. In addition, the calculated average properties of the $\gamma$-ray emission fairly agree with the measured ones as summarized in Table I.

Concerning the high-energy $\gamma$-ray transition of $E_{\gamma}>$ $10 \mathrm{MeV}$, such a transition is allowed only when the initial and final states have similar spin values. If the primary 


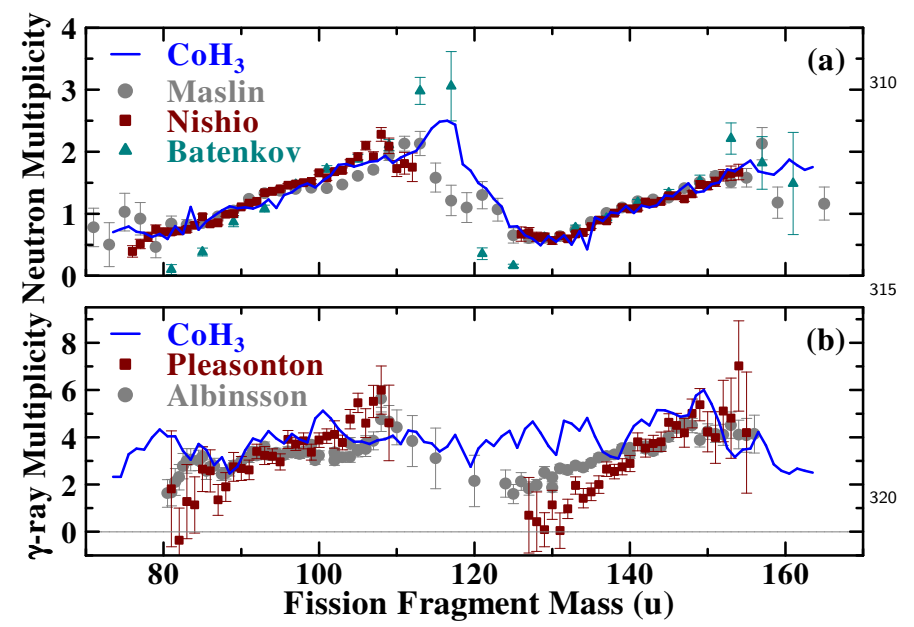

FIG. 4. Average prompt neutron multiplicity $\bar{\nu}(A)$ (a) and $\gamma$-ray multiplicity $\bar{M}_{\gamma}(A)$ (b) for ${ }^{235} \mathrm{U}\left(n_{\mathrm{th}}, f\right)$ from each $\mathrm{FF}_{325}$ calculated by the $\mathrm{CoH}_{3}$ code (solid lines). The $\bar{\nu}(A)$ are compared with the experimental data by Maslin et al. (circles) 60, Nishio et al. (rectangles) 43, and Batenkovet al. (triangles) 61. The $\bar{M}_{\gamma}(A)$ are compared with those by Albinsson et al. (circles) [59] and Pleasonton et al. (rectangles) 8 .

FFs are created with large angular momentum as mentioned above $(\bar{j}=6-8 \hbar)$, the transition must feed into the contributions to the spectrum at $E_{\gamma}>10 \mathrm{MeV}$ are given only by specific FFs, such as ${ }^{86} \mathrm{Br},{ }^{102,103} \mathrm{Nb}$, and ${ }^{109} \mathrm{Tc}$, as shown in the inset (b) of Fig. 2. In ${ }^{86} \mathrm{Br}$, for example, high-spin states up to $J=7$ are known at $E_{x} \leq$ $2852 \mathrm{MeV}$ [58], which are attributed to the configurations ${ }^{340}$ involving high- $j$ proton orbitals 62 . On the other hand, the maximum spin value reported in ${ }^{85} \mathrm{Br}$ was $J=7 / 2$ at $E_{x} \leq 4 \mathrm{MeV}$ [58], thus neutron emission from ${ }^{86} \mathrm{Br}$ is hindered. Such an individual feature on the low-lying states d be smeared out when one uses the systematics for the $\sigma^{2}$ parameter through all the possible FFs, obscuring ${ }^{345}$ the effects of high-spin states for particular FFs. This is demonstrated in Fig. 2, where the PFGS calculated using the systematics (dashed lines) become softer than the original calculation (solid lines). A better agreement for the high-energy part of the PFGS would be reached, ${ }^{350}$ if one unveils high-spin states at low excitation energies for some specific FFs, not observed so far. For example, a sudden onset of deformation, as observed in the $Z \sim 40$,

300 $N \sim 60$ and $Z \sim 64, N \sim 90$ regions 63, would increase the density of high-spin states due to the appearance of ${ }^{355}$ collective bands.

To understand the origin of the local bumps around $E_{\gamma}=4.0$ and $5.8 \mathrm{MeV}$, we also calculated the mass-gated the experimental mass resolution was taken into account. The calculations agree well with the measured spectra. In the analysis it was found that FFs in the vicinity

of the shell closures, ${ }^{132,133} \mathrm{Sn}$ and ${ }^{133,134} \mathrm{Sb}$, contribute to the bump at $E_{\gamma}=4.0 \mathrm{MeV}$. This is associated with the transition between the discrete levels in ${ }^{132} \mathrm{Sn}\left(E_{\gamma}\right.$ $=4.0$ and $4.4 \mathrm{MeV}$ ) and ${ }^{133} \mathrm{Sb}(4.2,4.3$, and $4.6 \mathrm{MeV})$. Interestingly, the complementary light FFs with $A \sim 100$, such as ${ }^{101,102} \mathrm{Zr}$, mostly contribute to the bump at $E_{\gamma}$ $=5.8 \mathrm{MeV}$ as shown in Fig. 3(b). We found that this is attributed to the transition in the residual nuclei left after one-neutron evaporation. Such a transition is allowed only when the primary FF has an excitation energy higher than $\sim 13 \mathrm{MeV}$, i.e., the sum of the energy released by neutron $(6 \sim 8 \mathrm{MeV})$ and $\gamma$ ray $(\sim 6 \mathrm{MeV})$. We note that in ${ }^{252} \mathrm{Cf}(\mathrm{sf})$ [20] the PFGS obtained in the mass region $A_{\mathrm{L}} \leq 114$ do not show any local structures. This is because the FFs with $A_{\mathrm{L}} \sim 100$ for ${ }^{252} \mathrm{Cf}(\mathrm{sf})$ have lower excitation energy of $\bar{E}_{x} \sim 12 \mathrm{MeV}$ [15].

Conclusion. In summary, we have measured the PFGS for ${ }^{235} \mathrm{U}\left(n_{\mathrm{th}}, f\right)$ up to energies of about $20 \mathrm{MeV}$. Our measurement extended the currently known $\gamma$-ray spectrum limit to higher energies by approximately a factor of two by gaining a factor of about $10^{5}$ in statistics compared to the measurements so far. The statistical Hauser-Feshbach model was applied to calculate the PFGS by considering the competition between neutron and $\gamma$-ray emissions for each FF. The calculated results showed that a limited number of light FFs, such as ${ }^{109} \mathrm{Tc}$, can emit high-energy $\gamma$-rays of $E_{\gamma}>10 \mathrm{MeV}$. Still the calculation underestimates the spectrum in this highenergy region. The reason might be the insufficient data for high-spin states at low excitation energies for some other FFs, required to allow the high-energy $\gamma$-ray transition from the primary FFs. It was also found that the bump structure at $E_{\gamma}=4.0 \mathrm{MeV}$ is created by the heavy FFs in the vicinity of the shell closures at $Z=50$ and $N$ $=82$. On the contrary, the $5.8-\mathrm{MeV}$ bump was suggested to originate mostly from the light FFs with $A \sim 100$.

The authors thank useful discussions with Drs A. N. Andreyev of University of York, and I. Nishinaka of National Institutes for Quantum and Radiological Science and Technology. We also thank the services of the ILL for the support during the measurement of the PFGS for ${ }^{235} \mathrm{U}\left(n_{\mathrm{th}}, f\right)$. The staff of the JRC in Geel is gratefully acknowledged for making the ${ }^{235} \mathrm{U}$ target. The present study is the result of "Measurement of highenergy prompt gamma-rays in fission for surveillance detector to monitor criticality of fuel debris" entrusted to Japan Atomic Energy Agency (JAEA) by the Ministry of Education, Culture, Sports, Science and Technology of Japan (MEXT).

\footnotetext{
* makii.hiroyuki@jaea.go.jp

† nishio.katsuhisa@jaea.go.jp
} 

terer, P. Singer, M. Klemens, J. P. Theobald, M. Hesse,475 F. Gönnenwein, and H. Ploeg, Z. Phys. A 356, 299 (1996)

[21] D. Biswas, B. Nayak, M. Cinausero, B. Fornal, G. Viesti, D. Bazzacco, M. DePoli, D. Fabris, E. Fioretto, M. Lunardon, D. Napoli, G. Nebbia, G. Prete, C. Ur,480 and G. Vedovato, Eur. Phys. J. A 4, 343 (1999).

[22] S. J. Luke, C. A. Gossett, and R. Vandenbosch, Phys. Rev. C 44, 1548 (1991).

[23] H. van der Ploeg, J. C. S. Bacelar, A. Buda, C. R. Laurens, A. van der Woude, J. J. Gaardho/je, Z. Żelazny,485
G. van 't Hof, and N. Kalantar-Nayestanaki, Phys. Rev. C 52, 1915 (1995).

[24] D. Pandit, S. Mukhopadhyay, S. Bhattacharya, S. Pal, A. De, and S. Banerjee, Phys. Lett. B 690, 473 (2010)

[25] S. Lemaire, P. Talou, T. Kawano, M. B. Chadwick, and D. G. Madland, Phys. Rev. C 73, 014602 (2006)

[26] J. Randrup and R. Vogt, Phys. Rev. C 80, 024601 (2009)

[27] R. Vogt, J. Randrup, D. A. Brown, M. A. Descalle, and W. E. Ormand, Phys. Rev. C 85, 024608 (2012)

[28] R. Vogt and J. Randrup, Phys. Rev. C 87, 044602 (2013)

[29] B. Becker, P. Talou, T. Kawano, Y. Danon, and I. Stetcu, Phys. Rev. C 87, 014617 (2013).

[30] I. Stetcu, P. Talou, T. Kawano, and M. Jandel, Phys. Rev. C 90, 024617 (2014).

31 O. Litaize, O. Serot, and L. Berge, Eur. Phys. J. A 51, 177 (2015)

[32] K.-H. Schmidt, B. Jurado, C. Amouroux, and C. Schmitt, Nucl. Data Sheets 131, 107 (2016), special Issue on Nuclear Reaction Data.

[33] P. Talou, T. Kawano, I. Stetcu, J. P. Lestone, E. McKigney, and M. B. Chadwick, Phys. Rev. C 94, 064613 (2016)

[34] R. Vogt and J. Randrup, Phys. Rev. C 96, 064620 (2017).

[35] P. Jaffke, P. Möller, P. Talou, and A. J. Sierk, Phys. Rev. C 97, 034608 (2018)

[36] K.-H. Schmidt and B. Jurado, Rep. Prog. Phys. 81, $106301(2018)$

[37] O. Litaize, A. Chebboubi, O. Serot, L. Thulliez, T. Materna, and M. Rapala, EPJ Nuclear Sci. Technol. 4, 28 (2018).

[38] C. Budtz-Jørgensen and H.-H. Knitter, Nucl. Phys. A 490, 307 (1988)

[39] D. J. Hofman, B. B. Back, C. P. Montoya, S. Schadmand, R. Varma, and P. Paul, Phys. Rev. C 47, 1103 (1993).

[40] T. Kawano, P. Talou, I. Stetcu, and M. Chadwick, Nucl. Phys. A 913, 51 (2013).

[41] H. Abele, D. Dubbers, H. Hase, M. Klein, A. Knopfler, M. Kreuz, T. Lauer, B. Markisch, D. Mund, V. Nesvizhevsky, A. Petoukhov, C. Schmidt, M. Schumann, and T. Soldner, Nucl. Instrum. Methods A 562, 407 (2006)

[42] H. Makii, K. Nishio, K. Hirose, R. Orlandi, R. Lguillon, T. Ogawa, T. Soldner, F.-J. Hambsch, M. Aïche, A. Astier, S. Czajkowski, R. Frost, S. Guo, U. Köster, L. Mathieu, T. Ohtsuki, C. Petrache, A. Pollitt, S. Sekimoto, K. Takamiya, and I. Tsekhanovich, Nucl. Instrum. Methods A 906, 88 (2018).

[43] K. Nishio, Y. Nakagome, H. Yamamoto, and I. Kimura, Nucl. Phys. A 632, 540 (1998)

[44] T. Kawano, P. Talou, M. B. Chadwick, and T. Watanabe, J. Nucl. Sci. Technol. 47, 462 (2010).

[45] S. Schmitt, J. Inst. 7, T10003 (2012).

[46] F.-J. Hambsch, private communication.

[47] S. Okumura, T. Kawano, P. Jaffke, P. Talou, and S. Chiba, J. Nucl. Sci. Technol. 55, 1009 (2018)

[48] A. C. Wahl, los Alamos National Laboratory Report No. LA-13928, 2002 (unpublished).

[49] T. Osawa, in Proceeding of Coordinated Meeting on $\mathrm{Nu}$ clear Data for Neutron Emission in the Fission Process, INDC(NDS)-251 (1991) pp. 71-80.

[50] T. Ohsawa, T. Horiguchi, and H. Hayashi, Nucl. Phys. A 653, 17 (1999)

51] T. Ohsawa, T. Horiguchi, and M. Mitsuhashi, Nucl. Phys. A 665, 3 (2000). 
[52] A. Koning and J. Delaroche, Nucl. Phys. A 713, 231 (2003).

[53] J. Kopecky and M. Uhl, Phys. Rev. C 41, 1941 (1990)

490

[54] M. Herman, R. Capote, M. Sin, A. Trkov, B. Carlson, P. Obložinsky, C. Mattoon, H. Wienke, S. Hoblit, Y.-S.510 Cho, G. Nobre, V. Plujko, and V. Zerkin, EMPIRE-3.2 - Malta Modular system for nuclear reaction calculations and nuclear data evaluation, User's Manual INDC-0603

495

[55] M. R. Mumpower, T. Kawano, J. L. Ullmann,515 M. Krtička, and T. M. Sprouse, Phys. Rev. C 96, 024612 (2017)

56] A. Gilbert and A. G. W. Cameron, Can. J. of Phys. 43, $1446(1965)$

57] T. Kawano, S. Chiba, and H. Koura, J. Nucl. Sci. Tech-;20 nol. 43, 1 (2006).

[58] R. Capote, M. Herman, P. Obložinský, P. Young, S. Goriely, T. Belgya, A. Ignatyuk, A. Koning, S. Hilaire, V. Plujko, M. Avrigeanu, O. Bersillon, M. Chad- wick, T. Fukahori, Z. Ge, Y. Han, S. Kailas, J. Kopecky, V. Maslov, G. Reffo, M. Sin, E. Soukhovitskii, and P. Talou, Nucl. Data Sheets 110, 3107 (2009)

[59] H. Albinsson and L. Lindow, aktiebolaget Atomenergi Studsvik(Nyköping, Sweden) Technical Report No. AE398, 1970 (unpublished).

[60] E. E. Maslin, A. L. Rodgers, and W. G. F. Core, Phys. Rev. 164, 1520 (1967).

[61] O. A. Batenkov, G. A. Boykov, F. Hambsch, J. H. Hamilton, V. A. Jakovlev, V. A. Kalinin, A. B. Laptev, V. E. Sokolov, and A. S. Vorobyev, AIP Conf. Proc. 769, 1003 (2005)

[62] M. G. Porquet, A. Astier, T. Venkova, I. Deloncle, F. Azaiez, A. Buta, D. Curien, O. Dorvaux, G. Duchêne, B. J. P. Gall, F. Khalfallah, I. Piqueras, M. Rousseau, M. Meyer, N. Redon, O. Stézowski, R. Lucas, and A. Bogachev, Eur. Phys. J. A 40, 131 (2009).

[63] K. Heyde and J. L. Wood, Rev. Mod. Phys. 83, 1467 (2011) 